\title{
Numerical Distribution Functions for Seasonal Unit
}

\section{Root Tests}

\author{
Ignacio Díaz-Emparanza* \\ University of the Basque Country, UPV/EHU \\ Address: Avenida Lehendakari Aguirre, 83. E48015 Bilbao, Spain. \\ Phone: +34 946013732, FAX: +34946013754 \\ Email: ignacio.diaz-emparanza@ehu.es
}

December 16, 2011

\begin{abstract}
When working with time series data observed at intervals smaller than a year, it is often necessary to test for the presence of seasonal unit roots. One of the most widely used methods for testing seasonal unit roots is that of HEGY, which provides test statistics with non-standard distributions. This paper describes a generalisation of this method for any periodicity and uses a response surface regressions approach to calculate the critical values and $P$ values of the HEGY statistics whatever the periodicity and sample size of the data. The algorithms are prepared with the Gretl open source econometrics package and some new tables of critical values for daily, hourly and halfhourly data are presented.
\end{abstract}

Keywords: seasonality, unit roots, surface response analysis

*Financial support from research project ECO2010-15332 from Ministerio de Ciencia e Innovación, and Econometrics Research Group IT-334-07 from the Basque Government are gratefully acknowledged. The SGI/IZO-SGIker UPV/EHU is gratefully aknowledged for its generous allocation of computational resources. The idea for this work emerged in a conversation with Riccardo 'Jack' Lucchetti. 


\section{Introduction}

Unit roots may cause severe problems in a regression model if they are not properly dealt with: this may imply inconsistent coefficient estimators and nonstandard distributions for significance tests and for forecast intervals. There have been many papers on testing for unit roots since the book by Fuller (1976), which introduced the test currently known as the Augmented Dickey-Fuller test, ADF (See also Dickey \& Fuller 1981). Apart from the ADF test, other tests worth mentioning are those by Phillips \& Perron (1988), the KPSS test for stationarity by Kwiatkowski et al. (1992) and the ADF-GLS test by Elliott, Rothenberg \& Stock (1996) which have become widely used by empirical economists. However, when working with time series data observed at intervals shorter than a year, the presence of unit roots should be tested for, not only in the long run (frequency $\omega=0$ ) but also in seasonal cycles. Over the last thirty years various methods have been proposed for testing for seasonal unit roots. For example, Hasza \& Fuller (1982) and Dickey, Hasza $\&$ Fuller (1984), proposed joint tests for all seasonal unit roots, but then Osborn, Chui, Smith \& Birchenhall (1988) and in particular Hylleberg, Engle, Granger \& Yoo (1990) (hereinafter HEGY) proposed tests that enable each of the seasonal, and frequency zero roots to be considered separatelly. There are also interesting tests of seasonal stability by Canova \& Hansen (1995), which also consider each frequency individually. The HEGY tests are not very difficult to implement, and have therefore become widely used among empirical economists.

One of the problems with most of the unit root tests mentioned above is that their statistics have non-standard distributions, so in practice one needs to interpolate the values in the tables published to compare them with the values calculated or simulate the empirical distributions for exactly the same model and the same sample size that is being used. MacKinnon (1994), uses simulation methods and surface response regressions to estimate the asymptotic distributions of a large 
number of unit roots and cointegration tests at zero frequency (long run). Then in MacKinnon (1996) he extends these results, providing a way to approximate small sample distributions too.

Harvey \& van Dijk (2006) apply the method of MacKinnon, using surface response regressions, to provide a simple way of obtaining critical values and $P$ values for any sample size and any order of lags of the endogenous variable in the regression for the HEGY tests already mentioned. All this, as in the original HEGY article, is for quarterly data.

The main objective of this paper is to obtain a generalisation of the method of Harvey and van Dijk for calculating the critical values and $P$ values of the HEGY statistics whatever the periodicity, $s$, and sample size $T$ of the data.

\section{Seasonal Unit Roots}

Hylleberg et al. (1990) study how to test for unit roots in seasonal time series. They take quarterly periodicity of data $(s=4)$ as their reference and assume that the series $x_{t}$ is generated by a possibly infinite order autoregressive process,

$$
\phi(L) x_{t}=\varepsilon_{t}
$$

where $L$ is the lag operator, such that $L x_{t}=x_{t-1}, \phi(L)$ represents the polynomial $1-\phi_{1} L-\phi_{2} L^{2}-\cdots$ and $\varepsilon_{t}$ is a white noise process with variance $\sigma_{\varepsilon}^{2}$.

To test the hypothesis that the roots of $\phi(L)=0$ are on the unit circle against the alternative hypothesis that are outside of the unit circle, they set up the following procedure. They show that equation (1) can be written in an equivalent form as:

$$
\phi^{*}(L) y_{4 t}=\pi_{1} y_{1 t-1}+\pi_{2} y_{2 t-1}+\pi_{3} y_{3 t-2}+\pi_{4} y_{3 t-1}+\varepsilon_{t}
$$


where

$$
\begin{aligned}
& y_{1 t}=\left(1+L+L^{2}+L^{3}\right) x_{t}=S(L) x_{t}, \\
& y_{2 t}=-\left(1-L+L^{2}-L^{3}\right) x_{t}, \\
& y_{3 t}=-\left(1-L^{2}\right) x_{t}, \\
& y_{4 t}=\left(1-L^{4}\right) x_{t}=\Delta_{4} x_{t}
\end{aligned}
$$

and $\phi^{*}(L)$ is a polynomial with all the roots outside the unit circle. Equation (2) may be estimated, in a consistent way, by ordinary least squares (OLS).

Testing the hypothesis of a unit root at zero frequency is equivalent to testing the significance of the coefficient $\pi_{1}$, and this can be done by using a $t$ statistic. However, this statistic does not follow a Student's $t$-distribution. Hylleberg et al. (1990) show that the asymptotic distribution of this $t_{\pi_{1}}$ statistic is the same as that of the Dickey-Fuller statistic.

The existence of a unit root at the Nyquist frequency, $\omega=\pi$, can be checked by a significance test on the coefficient $\pi_{2}$, also using a t-statistic. The presence of unit roots at frequencies $\omega= \pm \pi / 2$ can be tested by an F-statistic for the joint hypothesis that $\pi_{3}=\pi_{4}=0$.

Beaulieu \& Miron (1993) (and in a slightly different way Franses (1990)) study the problem of testing for seasonal unit roots with monthly data. They show that an equation with a similar structure to that of HEGY could also be proposed for this case. In the quarterly case there are two seasonal cycles at frequencies $\pm \pi / 2$ and $\pi$, but in the monthly case there are six seasonal cycles at frequencies $\pm \pi / 6, \pm \pi / 3, \pm \pi / 2, \pm 2 \pi / 3, \pm 5 \pi / 6$, and $\pi$ which, as Beaulieu and Miron show, makes the structure of the equation a little more complicated, as it depends on 12 filters instead of only 4 (See equations 4 and 5 in Beaulieu \& Miron 1993). There 
is also a version of equation (2) for weekly data (with $s=52$ ) in Cáceres (1996), which depends on 52 seasonal filters.

If you want to test for seasonal unit roots in an applied study, and you have quarterly data, then you are very lucky. You can use the aforementioned procedure of Harvey and van Dijk to calculate critical values or $P$-values for your tests. If you are working with monthly or weekly data you are not quite so lucky: you can use the tables of critical values in the articles by Beaulieu and Miron or Cáceres mentioned above, and use an interpolation method to calculate the critical values that you need, but as far as I know you do not have at your disposal any method for obtaining $P$-values. If you are working with daily data ( $s=5,6$ or 7 days a week), hourly data ( $s=24$ hours a day), etc. you are completely out of luck, you do not even have tables to compare with. As stated above, the main goal of this paper is to solve this problem.

The first step towards obtaining a general solution to the problem is to show a generalisation of equation (2) for any periodicity $s$. Such a generalisation is presented in the next section.

\section{Methodology}

A time series whose observations are regularly collected $s$ times a year ${ }^{1}$ can contain $[s / 2]$ different seasonal cycles, denoting by $[\cdot]$ the integer part of the number contained in brackets, i.e.

$$
[s / 2]=\left\{\begin{array}{lll}
s / 2 & \text { if } & \mathrm{s} \text { is even } \\
(s-1) / 2 & \text { if } & \mathrm{s} \text { is odd }
\end{array}\right.
$$

\footnotetext{
${ }^{1}$ It is sometimes convenient to change the reference period from one year, for example, to one day if data are hourly, $s=24$, or one week if data are daily, $s=7$.
} 
The angular frequencies corresponding to the seasonal cycles are $\omega_{j}=\frac{2 \pi j}{s}$, with $j=1, \ldots,[s / 2]$.

Let $y_{t}$ be a time series integrated of order one at frequencies $\omega_{1}, \ldots, \omega_{q} \in$ $[0, \pi]$, and let its autoregressive representation be

$$
\phi(L) y_{t}=\gamma^{\prime} D_{t}+u_{t}
$$

where $\phi(L)$ is a polynomial on the lag operator, $D_{t}$ is a column vector with deterministic terms, $\gamma$ is its associated coefficients vector and $u_{1}, \ldots, u_{T}$ are $i i d\left(0, \sigma_{u}^{2}\right)$.

Now define $\mathrm{z}(L)=1-L^{s}$ as the polynomial of order $s$ made up of (single) unit roots $\theta_{1}, \ldots, \theta_{s}$ at frequencies ${ }^{2} \pm \omega_{1}, \ldots, \pm \omega_{q}$, being $q=[s / 2]+1$. The roots at frequencies 0 and $\pi$ (only present if $s$ is even) are real, but the rest have complex-conjugate values. With no loss of generality we will assume from now on that $s$ is even, and that $\theta_{1}=1$ (the root corresponding to $\omega_{1}=0$ ), $\theta_{2}=-1$ (the root of frequency $\omega_{2}=\pi$ ) and the following go in pairs so that $\theta_{j}$ and $\theta_{j+1}$ for $j=3,5,7 \ldots$ correspond to pairs of complex conjugate roots.

Model (3) may be expressed as (the proof is in appendix A):

$$
\begin{aligned}
\phi^{*}(L) \mathrm{z}(L) y_{t}= & \gamma^{\prime} D_{t}+\pi_{1} y_{1, t-1}+\pi_{2} y_{2, t-1}+ \\
& +\sum_{j=1}^{\frac{s}{2}-1}\left(\pi_{2 j+1} y_{2 j+1, t-1}+\pi_{2 j+2} y_{2 j+1, t-2}\right)+u_{t}
\end{aligned}
$$

where

$$
\begin{aligned}
y_{1 t} & =\frac{\mathrm{z}(L)}{1-L} L y_{t} \\
y_{2 t} & =-\frac{\mathrm{z}(L)}{1+L} L y_{t}
\end{aligned}
$$

\footnotetext{
${ }^{2}$ We have two unit roots per each $\omega_{j} \in(0, \pi)$ but only one per each $\omega_{j}=0, \pi$.
} 


$$
\begin{aligned}
y_{2 j+1, t} & =-\frac{\mathrm{z}(L)}{\delta_{2 j+1}(L) \delta_{2 j+2}(L)} y_{t} ; \quad j=1,2, \ldots,\left[\frac{s}{2}\right]-1 \\
\mathrm{z}(L) y_{t} & =\left(1-L^{s}\right) y_{t}
\end{aligned}
$$

being $\delta_{i}(L)=1-\theta_{i}^{-1} L$ for any $i=1, \ldots, s$.

In practice, using data for a given sample size $T, \phi^{*}(L)$ is approximated by a finite order $p$ polynomial, so that the model (4) may be written as

$$
\begin{aligned}
\mathrm{z}(L) y_{t}= & \gamma^{\prime} D_{t}+\pi_{1} y_{1, t-1}+\pi_{2} y_{2, t-1}+ \\
& +\sum_{j=1}^{\frac{s}{2}-1}\left(\pi_{2 j+1} y_{2 j+1, t-1}+\pi_{2 j+2} y_{2 j+1, t-2}\right)+ \\
& +\sum_{i=1}^{p} \phi_{i} \mathrm{z}(L) y_{t-i}+u_{t}
\end{aligned}
$$

It is important to note that if $s$ is odd the series does not have a seasonal root at the angular frequency $\omega=\pi$, so the term $\pi_{2} y_{2, t-1}$ will not appear in equations (4) and (5).

Filters at $y_{2 j+1, t}$ in the quarterly case reflect the same transformations of the HEGY paper, in the monthly case are equal to those of Beaulieu and Miron apart from a scale factor and for $s=52$ are exactly the same as those used by Cáceres.

In order to test for unit roots, (5) is estimated by OLS and then the significance of the coefficients is tested using appropiate finite sample distributions based on Monte Carlo results. $\pi_{1}=0$ implies that the series contains a unit root at the zero frequency. When $\pi_{2}=0$, there is a seasonal unit root at frequency $\pi$ (two observations per cycle). For $k>2$ and even, when $\pi_{k}=\pi_{k-1}=0$, seasonal unit roots are present at frequencies $\pm \omega_{\frac{k+2}{2}}$. For frequencies 0 and $\pi$ it suffices to examine the relevant $t$-statistics for $\pi_{k}=0$ against the alternative that $\pi_{k}<0$. For the other frequencies, the strategy is to test $\pi_{k}=\pi_{k-1}=0$, with $k>2$ and 
even. This may be done by means of two $t$-statistics, but an $F$-statistic for such hypothesis, referred to here as $F_{k, k-1}$, proves to be more powerful.

By means of Itô calculus, Phillips (1987) shows that $t_{\pi_{1}}$ and $t_{\pi_{2}}$ asymptotically have the Dickey-Fuller distribution. Ahtola \& Tiao (1987) and Chan \& Wei (1988) note that the asymptotic distributions of the odd $t$ statistics (apart from $t_{\pi_{1}}$ ) are the same across frequencies. Beaulieu and Miron show, using their Lemma 1, that the even $t$ statistics also have the same asymptotic distribution, and that the $F_{k, k-1}$ statistics, for $k$ even and $k>2$ converge in law to $\frac{1}{2}\left(t_{\pi_{k}}^{2}+t_{\pi_{k-1}}^{2}\right)$. Because all odd t-statistics (apart from $t_{\pi_{1}}$ ) have the same distribution and all even statistics (apart from $t_{\pi_{2}}$ ) have the same distribution, all of the $F_{k, k-1}$ statistics, for $k=4,6,8, \ldots$, have the same asymptotic distribution. By analysing the proof in Chan \& Wei (1988) and lemmas 1 to 4 in the cited article by Beaulieu and Miron it can be seen that this asymptotic distribution does not depend on the periodicity of the data. This is the key feature of equation (5), which enables a general method to be set up that is valid for any periodicity. However, different periodicities imply a different number of regressors in this equation so a movement should be expected in the finite sample distributions of the $t$ and $F$ statistics depending on the periodicity $s$.

\section{Surface response analysis}

As with the ADF test of Dickey \& Fuller (1979), HEGY test statistics, have nonstandard distributions even asymptotically. I am aware only of tables of critical values for the tests for quarterly, monthly and weekly data. Anyone working, for example with periodicity 24 (hourly data), has no tables to compare with. Furthermore, even in the case of a periodicity for which tables are available, even though the asymptotic theory of these tests is well developed it is not easy for applied researchers to calculate the $P$-value of a given test statistic. Here I use a general- 
ization of Harvey and Van Dijk's procedure, based on response surface regressions, that serve to obtain $P$-values and critical values not only for quarterly data but for any periodicity.

To implement the response surface regressions, the first step was to estimate the relevant quantiles of the distributions of the HEGY tests for several combinations of T (effective sample size), $p$ (autoregressive order) and $s$ (periodicity) from a large set of Monte Carlo simulations and, following MacKinnon (2000), the process was then repeated $M$ times for each value of $T$ to obtain more accurate results. Each experiment consisted on $N$ replications, where the series $y_{t}$ was generated by the data generation process $\Delta_{s} y_{t}=u_{t}$ with $u_{t} \sim \operatorname{nid}(0,1)$ and the equation estimated was (5) with $p=0,1, \ldots, p_{\max }$ (being $p_{\max }$ the values in the table below) and two alternatives of the deterministic component: a) intercept and $s-1$ seasonal dummies, b) intercept, $s-1$ seasonal dummies and a linear trend.

All the different cases simulated are reported in table 1 . The first row of this table, for example, indicates that for quarterly data $(s=4)$, the model was simulated for $T=48,100,136,200$ and 400 observations of $y_{t}$, considering autoregressive orders from 0 to 8 , and using $N=50000$ replications and $M=25$ repetitions for each value of $T$. The last three columns of the table show the $95 \%$ accuracy, $A$, of the quantiles $0.10,0.05$ and 0.01 estimated by the simulation method, obtained by the following approximation (See, for example, Kleijnen 1987):

$$
A_{q} \approx 1.96 \sqrt{\frac{q(1-q)}{N M}}
$$

For example, the value for $A_{0.10}$ in the first row, 0.00053 , indicates that the estimation of the quantile 0.01 obtained by simulation with $N=50000$ and $M=$ 25 with a $95 \%$ confidence has an associated probability in the interval $(0.01 \pm$ $0.00053)=(0.00947,0.01053)$. 
The Monte Carlo simulations were programmed in Gretl 1.9.4 (See Cottrell \& Lucchetti 2011). From this version Gretl uses as random number generator for the uniform distribution the SIMD-oriented Fast Mersenne Twister (SFMT) (See Saito \& Matsumoto 2008) in particular the implementation referred to as SFMT19937, which has a period of $2^{19937}-1$ and is based on version 1.3 .3 of the original C code by Saito and Matsumoto ${ }^{3}$. Gretl uses Ziggurat (Marsaglia \& Tsang 2000) as the default method for generating normal variates on the basis of uniform input.

From each Monte Carlo experiment a record is made of the 221 estimated quantiles of the statistics $t_{\pi_{1}}, t_{\pi_{2}}$ and $F_{k, k-1}$ with $k=4,6,8, \ldots$, for probabilities $\alpha=0.0001,0.0002,0.0005,0.001,0.002, \ldots, 0.01,0.015, \ldots, 0.99,0.991, \ldots$ 0.999, 0.9995, 0.9998, 0.9999 and the estimated quantiles are used as dependent variables in response surface regressions of the form

$$
\begin{aligned}
q_{i}^{\alpha}(T, k)= & \theta_{\infty}^{\alpha}+\theta_{1}^{\alpha} \frac{1}{T}+\theta_{2}^{\alpha} \frac{1}{T^{2}}+\theta_{3}^{\alpha} \frac{1}{T^{3}}+\theta_{4}^{\alpha} \frac{k}{T}+\theta_{5}^{\alpha} \frac{k}{T^{2}}+\theta_{6}^{\alpha} \frac{k}{T^{3}}+ \\
& +\theta_{7}^{\alpha} \frac{k^{2}}{T}+\theta_{8}^{\alpha} \frac{k^{2}}{T^{2}}+\theta_{9}^{\alpha} \frac{k^{2}}{T^{3}}+\theta_{10}^{\alpha} \frac{k^{3}}{T}+\theta_{11}^{\alpha} \frac{k^{3}}{T^{2}}+\theta_{12}^{\alpha} \frac{k^{3}}{T^{3}}+ \\
& +\theta_{13}^{\alpha} \frac{s}{T}+\theta_{14}^{\alpha} \frac{s}{T^{2}}+\theta_{15}^{\alpha} \frac{s}{T^{3}}+e_{i}
\end{aligned}
$$

where $q_{i}^{\alpha}(T, k)$ denotes quantile $\alpha$ obtained from the experiment $i$-th with sample size $T$ and AR order $k$. This functional form was arrived at after some experimentation: is based on the one used by Harvey and van Dijk but includes a third degree in $k$, which is significant when $s>4$, and adds three terms to take periodicity into account. Parameter $\theta_{\infty}^{\alpha}$ represents quantile $\alpha$ of the asymptotic distribution when $T \rightarrow \infty$. In the regressions estimated, some of the coefficients were not significant but I prefered to maintain the same explanatory variables in all regressions, given that with 50000 or 10000 observations this does not imply much loss of efficiency

\footnotetext{
${ }^{3}$ This can be examined using the SourceForge 'viewvc' interface located at http://gretl.cvs.sourceforge.net/viewvc/gretl/gretl/rng/
} 
and the algorithms are thus much simpler.

When the paremeters of equation (6) are estimated by ordinary least squares, the errors are heteroscedastic with variance depending on $T$ and $s$. Several different alternatives of weighted least squares were used to take heteroscedasticity into account and in all cases the best result proved to be that of the default hsk native command of Gretl. The procedure implemented by this command involves three steps: first an OLS estimation of the model, then an auxiliary regression to generate an estimate of the error variance, then finally weighted least squares, using the reciprocal of the estimated variance as the weight. In the auxiliary regression the log of the squared residuals from the first OLS is regressed on the original regressors and their squares. The log transformation is performed to ensure that the estimated variances are non-negative.

For the monthly case $(s=12)$, Beaulieu \& Miron (1993, pp 316-317) say with respect to the $F_{k, k-1}$ statistics $^{4}$ for the different values of $k=4,6,8,10,12$ that "investigation of the finite sample distributions for a subset of the cases considered below indicates that these distributions are similar for a given number of simulations and converge as the number of simulations increases". I used equation (6) to test the hypothesis that all the $F_{k, k-1}$ statistics have the same distribution across different values of $k$. This is a joint test of the null hypothesis that parameters $\theta_{\infty}^{\alpha}, \theta_{1}^{\alpha}, \ldots, \theta_{15}^{\alpha}$ for the $F$ statistics are the same for different $k$. Assuming normality, albeit asymptotically, the statistic has a very high $P$-value so at a 5\% significance level the null is not rejected and the conclusion is that the distribution of $F_{k, k-1}$ does not depend on $k$ with finite samples either. The only remaining concerns are three distributions: $t_{\pi_{1}}, t_{\pi_{2}}$ and a generic $F$ which is the same for different values of $k$. So all the simulations of the different $F_{k, k-1}$ statistics can be used jointly for estimating the parameters of (6), thus improving the precission

\footnotetext{
${ }^{4}$ And for the $t_{\text {even }}, t_{\text {odd }}$ statistics as well.
} 
of the estimates. Specifically, there are $N([s / 2]-1)$ observations for estimating the quantiles of the $F$ statistic (for example in the monthly case, with $N=10000$ there are 50000 observations).

After the response surface regression (6) is estimated for all 221 quantiles for the three statistics, an interpolation between these values may be performed using the method of MacKinnon (1996). Consider the regression

$$
\Phi^{-1}(\alpha)=\gamma_{0}+\gamma_{1} \hat{q}(\alpha)+\gamma_{2} \hat{q}^{2}(\alpha)+\gamma_{3} \hat{q}^{3}(\alpha)+e_{\alpha}
$$

where $\alpha$ denotes one of the 221 points at which the quantiles are estimated, with $0<\alpha<1, \hat{q}(\alpha)$ denotes the estimate of $q^{\alpha}$ and $\Phi^{-1}(\alpha)$ is the inverse of the cumulative standard normal distribution function. There is enough empirical evidence to show that this equation may be a good candidate for approximating the distribution of a two-tailed test statistic, such as $t_{\pi_{1}}$ and $t_{\pi_{2}}$, in a small region around a specified value of $\alpha$. For an F-type test a $\chi^{2}(2)$ is a better option than the normal distribution in $\Phi^{-1}(\alpha)$. Equation (7) is usually estimated with a small, odd number of points, $\ell$, around the specified significance level, in particular, $\ell=9,11$, 13 or 15 points are considered reasonable ${ }^{5}$. To account for heteroscedasticity and serial correlation MacKinnon suggests employing a feasible GLS estimator using a symmetric covariance matrix with elements

$$
\hat{\omega}_{i j}=\operatorname{s.e}\left(\hat{\theta}_{\infty}^{\alpha_{i}}\right) \operatorname{s.e}\left(\hat{\theta}_{\infty}^{\alpha_{j}}\right) \sqrt{\frac{\alpha_{i}\left(1-\alpha_{j}\right)}{\alpha_{j}\left(1-\alpha_{i}\right)}}, \quad i<j
$$

where the standard errors of $\hat{\theta}_{\infty}^{\alpha_{i}}$ are obtained from the OLS estimation of equation (7).

In order to calculate the $P$-value for an observed test statistic, $\tau_{*}$, it is possible

\footnotetext{
${ }^{5}$ I see little difference in the results from 9 to 15 , so in the Gretl functions provided this parameter is left to the choice of the user.
} 
simply to estimate equation (7) for an small set of values of $\hat{q}(\alpha)$ near $\tau_{*}$ and then compute

$$
P^{*}=\Phi\left(\hat{\gamma}_{0}+\hat{\gamma}_{1} \tau_{*}+\hat{\gamma}_{2} \tau_{*}^{2}+\hat{\gamma}_{3} \tau_{*}^{3}\right)
$$

To calculate the critical values of the tests the following equation may be used

$$
\hat{q}(p)=\delta_{0}+\delta_{1} \Phi^{-1}(p)+\delta_{2}\left(\Phi^{-1}(p)\right)^{2}+\delta_{3}\left(\Phi^{-1}(p)\right)^{3}+e_{p}^{*}
$$

The method consists of first finding the quantile $p^{*}$ from the set of 221 mentioned above that is closest to the probability $p$ whose critical value is to be obtained, then estimating the $\delta$ coefficients in (10) with the $(\ell-1) / 2$ quantiles above and the $(\ell-1) / 2$ quantiles below $p^{*}$ and finally evaluating the right hand side of the regression estimated at $p$ to obtain the desired critical value.

\section{Implementation of the algorithms as Gretl functions}

Some scripts prepared by the author can be found at http://bit.Iy/ID-GHegy that contain:

- a function for obtaining the $P$-values of the $t_{\pi_{1}}, t_{\pi_{2}}$, and $F_{k, k-1}$ tests;

- a function that includes an algorithm for calculating the cumulative density functions of the three tests, i.e. obtaining critical values for a given probability;

- and finally a function for automatically calculating the HEGY tests and Pvalues for any periodicity. 


\section{Ensuring the quality of the computer algorithm and re- porting new tables of critical values}

Using the algorithm explained above the critical values for the same sample sizes reported in tables $1 \mathrm{~A}-1 \mathrm{~B}$ in the HEGY article, Table A.1 in the article by Beaulieu and Miron, and Table 3 in the paper by Cáceres were calculated. The results are shown here in Table 2.

A comparison of the critical values in the first part of this table and the tables in HEGY reveals very reasonable differences between. For the $t_{\pi_{1}}$ and $t_{\pi_{2}}$ statistics the differences decrease when the reported probability and the sample size increase. The biggest difference is 0.55 and the smallest is 0.05 . For the $F$ statistic, the differences increase with the reported probability and decrease with the sample size. The biggest difference is 1.14 and the smallest is 0.08 . Remember that the tables in HEGY were obtained by a single simulation with 24000 observations. The tables here were obtained with the use of the method decribed above, based on equation (10), using all the data resulting from simulations in Table 1 , and $M=25$ repetitions for each model. So, although both are estimations or approximations of the true critical values, a much lower variance and thus a more accurate approximation can be expected from the method described in this paper ${ }^{6}$.

A similar comparison between the values reported in Table A.1 in the article by Beaulieu and Miron and those of the second part of Table 2 here shows that the maximum divergence is 0.06 with the differences in $90 \%$ of the cells of the table being within an interval of \pm 0.03 . This appreciable better precission is because the $t$ tables in BM are also generated with 24000 observations, but by stacking them all 120000 were used for the $F$ test.

\footnotetext{
${ }^{6}$ The worse properties of the HEGY critical values are confirmed by the, somewhat erratic behaviour (sometimes decreasing with $T$ and sometimes increasing with $T$ ) of the values reported in their tables.
} 
Cáceres shows the critical values only for $T=468$ observations. He reports the distribution of $t_{\pi_{1}}, t_{\pi_{2}}$ and the $F_{k, k-1} \quad k=3,4 \ldots, 27$ statistics separately for each frequency (25 rows), without taking into account that the asymptotic distributions of these statistics for the different $k$ values are all the same. The differences between the values shown in the third part of Table 2 here and those reported by Cáceres for $t_{\pi_{1}}$ and $t_{\pi_{2}}$ are very small, and are all within the interval \pm 0.03 . The values for the $F$ tests are very similar but in this case the comparison is between values in one row in the present paper and values in 25 rows, so in some cases they are a little bigger, being in general contained in an interval of \pm 0.07 . This case is especially important because, as indicated in Table 1, I have not used periodicity $s=52$ in the simulations. In all cases the critical values are forecasts based on the estimated coefficients of regression (10), but in this particular case the forecast is made for a value for the periodicity that was not in the sample. It is striking to see that the results agreed so closely.

Furthermore, this comparisons serve to ensure that the algorithm prepared here does not work in the wrong direction because does not present major divergences with the tables published.

Given that this paper uses a much more accurate method for estimating critical values, it is not being over-bold to say that Table 2 here presents more reliable critical values than those in the original papers mentioned above.

On the other hand, it is very easy to apply the algorithm presented here to calculate critical value tables for other periodicities. Table 3 contains some critical values for the HEGY tests for three alternative sets of daily data: $s=5$ (one observation for each working day of the week), $s=6$ (e.g. every day except Sunday) and $s=7$ (one observation for each day of the week). Table 4 presents critical values for the HEGY tests for two sets of hourly data: $s=24$ (one observation per hour, with the day as the reference period), $s=24 \times 7=168$ (one observation 
per hour, with the week as the reference period). Finally, Table 5 shows the critical values for two cases of half-hourly data: $s=48$ (one observation per half-hour, with the day as the reference period), $s=48 \times 7=336$ (one observation per half-hour, with the week as reference period).

\section{Conclusion}

The HEGY $t$ and $F$ test statistics for seasonal unit roots have non-standard distributions that vary with the sample size, the number of autoregressive lags included in the model, the type and number of deterministic components and the periodicity of the data. Tables of critical values for the quarterly, monthly and weekly cases have already been published for some specific sample sizes, zero autoregressive lags, and several deterministic components.

A method based on surface regressions has also been published which calculates the $P$-values and critical values of these tests for quarterly data for any sample size and autoregressive order.

In the present article, I extend this method so that $P$-values and critical values can be obtained also for any periodicity. Lemmas 1 and 4 in Beaulieu \& Miron (1993) are the theoretical basis that enables it to be determined that the $F$ statistics for frequencies in $(0, \pi)$ have equal asymptotic distributions. This result enables a general algorithm to be set up for obtaining critical values and $P$-values for any periodicity.

In Section 4 the procedure for estimating the surface regresions based on Monte Carlo simulations and for obtaining the $P$-values and critical values of the different statistics is explained in detail. Users can find Gretl scripts for applying these techniques in empirical analyses at http://bit. Iy/ID-GHegy

Based on the algorithm for calculating critical values, Section 6 presents some 
tables for the cases already known (quarterly, monthly and weekly data) and also new tables for periodicities whose critical values were not hitherto available.

\section{A Appendix}

To obtain the representation (4) of $y_{t}$, start from equation (3) and use the following transformation [c.f. the univariate and the seasonal multivariate case in Hylleberg et al. (1990)]

$$
\phi(L)=\sum_{k=1}^{s} \lambda_{k} \frac{\mathrm{z}(L)}{\delta_{k}(L)}+\mathrm{z}(L) \phi^{* *}(L)
$$

where

$$
\delta_{k}(L)=1-\theta_{k}^{-1} L ; \quad \lambda_{k}=\frac{\phi\left(\theta_{k}\right)}{\prod_{j \neq k} \delta_{j}\left(\theta_{k}\right)}
$$

and $\phi^{* *}(L)$ is a polinomial with all its roots outside the unit circle.

Alternatively, (11) may be written as

$$
\phi(L)=\sum_{k=1}^{s} \lambda_{k} \frac{\mathrm{z}(L)}{\delta_{k}(L)}\left[1-\delta_{k}(L)\right]+\mathrm{z}(L) \phi^{*}(L)
$$

where $\phi^{*}(L)=\phi^{* *}(L)+\sum_{k=1}^{s} \theta_{k} \cdot I$.

Now rearrange to avoid complex coefficients and simplify expression (13). This equation is written as

$$
\begin{aligned}
\phi(L)= & \lambda_{1} \frac{\mathrm{z}(L)}{1-L} L+\lambda_{2} \frac{\mathrm{z}(L)}{1+L}(-L)+ \\
& +\sum_{j=1}^{\frac{s}{2}-1}\left\{\lambda_{2 j+1} \frac{\mathrm{z}(L)}{\delta_{2 j+1}(L) \delta_{2 j+2}(L)}\left[1-\delta_{2 j+1}(L)\right] \delta_{2 j+2}(L)+\right. \\
& \left.+\lambda_{2 j+2} \frac{\mathrm{z}(L)}{\delta_{2 j+1}(L) \delta_{2 j+2}(L)}\left[1-\delta_{2 j+2}(L)\right] \delta_{2 j+1}(L)\right\}+ \\
& +\mathrm{z}(L) \phi^{*}(L)
\end{aligned}
$$




\section{Given that}

$$
\begin{gathered}
\lambda_{2 j+1}\left[1-\delta_{2 j+1}(L)\right] \delta_{2 j+2}(L)+\lambda_{2 j+2}\left[1-\delta_{2 j+2}(L)\right] \delta_{2 j+1}(L)= \\
=\lambda_{2 j+1}\left[\delta_{2 j+2}(L)-\delta_{2 j+1}(L) \delta_{2 j+2}(L)\right]+\lambda_{2 j+2}\left[\delta_{2 j+1}(L)-\delta_{2 j+2}(L) \delta_{2 j+1}(L)\right]= \\
=\lambda_{2 j+1}\left[1-\frac{1}{\theta_{2 j+2}} L-\left(1-\frac{1}{\theta_{2 j+1}} L\right)\left(1-\frac{1}{\theta_{2 j+2}} L\right)\right]+ \\
+\lambda_{2 j+2}\left[1-\frac{1}{\theta_{2 j+1}} L-\left(1-\frac{1}{\theta_{2 j+1}} L\right)\left(1-\frac{1}{\theta_{2 j+2}} L\right)\right]= \\
=\lambda_{2 j+1}\left(\frac{1}{\theta_{2 j+1}} L-\frac{1}{\theta_{2 j+1} \theta_{2 j+2}} L^{2}\right)+\lambda_{2 j+2}\left(\frac{1}{\theta_{2 j+2}} L-\frac{1}{\theta_{2 j+1} \theta_{2 j+2}} L^{2}\right)= \\
=\lambda_{2 j+1}\left(\frac{1}{\theta_{2 j+1}} L-L^{2}\right)+\lambda_{2 j+2}\left(\frac{1}{\theta_{2 j+2}} L-L^{2}\right)
\end{gathered}
$$

Then polinomial $\phi(L)$ may be expressed as:

$$
\begin{aligned}
\phi(L)= & \lambda_{1} \frac{\mathrm{z}(L)}{1-L} L+\lambda_{2} \frac{\mathrm{z}(L)}{1+L}(-L)+ \\
& +\sum_{j=1}^{\frac{s}{2}-1} \frac{\mathrm{z}(L)}{\delta_{2 j+1}(L) \delta_{2 j+2}(L)}\left[\left(\lambda_{2 j+1} \frac{1}{\theta_{2 j+1}}\right) L-\left(\lambda_{2 j+1}+\lambda_{2 j+2}\right) L^{2}\right]+ \\
& +\mathrm{z}(L) \phi^{*}(L)
\end{aligned}
$$

If we define a new set of real parameters $\pi_{1}, \pi_{2}, \ldots$ such that:

$$
\left.\begin{array}{c}
\lambda_{1}=-\pi_{1} \\
\lambda_{2}=-\pi_{2} \\
\lambda_{2 j+1} \frac{1}{\theta_{2 j+1}}+\lambda_{2 j+2} \frac{1}{\theta_{2 j+2}}=\pi_{2 j+1} \\
-\left(\lambda_{2 j+1}+\lambda_{2 j+2}\right)=\pi_{2 j+2}
\end{array}\right\} \quad j=1,2, \ldots, \frac{s}{2}-1
$$

Then we have that

$$
\begin{aligned}
\phi(L)= & -\pi_{1} \frac{\mathrm{z}(L)}{1-L} L+\pi_{2} \frac{\mathrm{z}(L)}{1+L}(-L)+ \\
& +\sum_{j=1}^{\frac{s}{2}-1} \frac{\mathrm{z}(L)}{\delta_{2 j+1}(L) \delta_{2 j+2}(L)}\left[\pi_{2 j+1} L+\pi_{2 j+2} L^{2}\right]+ \\
& +\mathrm{z}(L) \phi^{*}(L)
\end{aligned}
$$


Then, equation (3) can be rewritten as

$$
\begin{aligned}
& -\pi_{1} \frac{\mathrm{z}(L)}{1-L} L y_{t}+\pi_{2} \frac{\mathrm{z}(L)}{1+L} L y_{t}+\sum_{j=1}^{\frac{s}{2}-1}\left[\pi_{2 j+1} \frac{\mathrm{z}(L)}{\delta_{2 j+1}(L) \delta_{2 j+2}(L)} L y_{t}+\right. \\
& \left.+\pi_{2 j+2} \frac{\mathrm{z}(L)}{\delta_{2 j+1}(L) \delta_{2 j+2}(L)} L^{2} y_{t}\right]+\mathrm{z}(L) \phi^{*}(L) y_{t}=\gamma^{\prime} D_{t}+u_{t}
\end{aligned}
$$

and reordering the terms the following is obtained

$$
\begin{aligned}
\phi^{*}(L) \mathrm{z}(L) y_{t}= & \gamma^{\prime} D_{t}+\pi_{1} \frac{\mathrm{z}(L)}{1-L} L y_{t}-\pi_{2} \frac{\mathrm{z}(L)}{1+L} L y_{t}- \\
& -\sum_{j=1}^{\frac{s}{2}-1}\left[\pi_{2 j+1} \frac{\mathrm{z}(L)}{\delta_{2 j+1}(L) \delta_{2 j+2}(L)} L y_{t}+\pi_{2 j+2} \frac{\mathrm{z}(L)}{\delta_{2 j+1}(L) \delta_{2 j+2}(L)} L^{2} y_{t}\right]+ \\
& +\mathrm{z}(L) \phi^{*}(L) y_{t}+u_{t}
\end{aligned}
$$

If we call

$$
\begin{aligned}
y_{1 t} & =\frac{\mathrm{z}(L)}{1-L} L y_{t} \\
y_{2 t} & =-\frac{\mathrm{z}(L)}{1+L} L y_{t} \\
y_{2 j+1, t} & =-\frac{\mathrm{z}(L)}{\delta_{2 j+1}(L) \delta_{2 j+2}(L)} ; j=1,2, \ldots, \frac{s}{2}-1 \\
\mathrm{z}(L) y_{t} & =\left(1-L^{s}\right)
\end{aligned}
$$

The previous equation can be written as

$$
\begin{aligned}
\phi^{*}(L) \mathrm{z}(L) y_{t}= & \gamma^{\prime} D_{t}+\pi_{1} y_{1, t-1}+\pi_{2} y_{2, t-1}+ \\
& +\sum_{j=1}^{\frac{s}{2}-1}\left[\pi_{2 j+1} y_{2 j+1, t-1}+\pi_{2 j+2} y_{2 j+1, t-2}\right]+u_{t}
\end{aligned}
$$




\section{References}

Ahtola, J. A. \& Tiao, G. C. (1987), 'Distribution of least squares estimators of autoregressive parameters for a process with complex roots on the unit circle', Journal of Time Series Analysis 8, 1-14.

Beaulieu, J. \& Miron, J. (1993), 'Seasonal unit roots in aggregate U.S. data', Journal of Econometrics 54, 305-28.

Canova, F. \& Hansen, B. E. (1995), 'Are seasonal patterns constant over time? a test for seasonal stability', Journal of Business and Economic Statistics 13, 237252.

Chan, N. H. \& Wei, C. Z. (1988), 'Limiting distributions of least squares estimates of unstable autoregressive processes', Annals of Statistics 16, 367-401.

Cottrell, A. \& Lucchetti, R. (2011), Gretl Command Reference, Department of Economics, Wake Forest University. http://gretl.sourceforge.net (accessed on sep, 5, 2011).

Cáceres, J. (1996), ‘Contraste de raíces unitarias en datos semanales', Estadística Española 38(141), 139-159.

Dickey, D. \& Fuller, W. (1979), 'Distribution of the estimators for autoregressive time series with a unit root', Journal of the American Statistical Association 74, 427-31.

Dickey, D. \& Fuller, W. (1981), 'Likelihood ratio statistics for autoregressive time series with a unit root', Econometrica 49, 1057-1071.

Dickey, D., Hasza, D. \& Fuller, W. (1984), 'Testing for unit roots in seasonal time series', Journal of American Statistical Association 79, 355-67. 
Elliott, G., Rothenberg, T. J. \& Stock, J. H. (1996), 'Efficient tests for an autoregressive unit root', Econometrica 64(4), 813-36.

Franses, P. (1990), Testing for seasonal unit roots in monthly data, Technical Report 9032, Econometric Institute.

Fuller, W. (1976), Introduction to Statistical Time Series, Jonh Wiley.

Harvey, D. I. \& van Dijk, D. (2006), 'Sample size, lag order and critical values of seasonal unit root tests', Computational Statistics \& Data Analysis 50, 27342751. available at http://www.sciencedirect.com.

Hasza, D. \& Fuller, W. (1982), ‘Testing for nonstationary parameter specifications in seasonal time series models', The Annals of Statistics 10, 1209-1216.

Hylleberg, S., Engle, R. F., Granger, C. W. J. \& Yoo, B. S. (1990), 'Seasonal integration and cointegration', Journal of Econometrics 44, 215-38.

Kleijnen, J. P. C. (1987), Statistical Tools for Simulation Practitioners, Marcel Dekker, Inc., New York.

Kwiatkowski, D., Phillips, P., Schmidt, P. \& Shin, Y. (1992), 'Testing the null hypothesis of stationarity against the alternative of a unit root: How sure are we that economic time series have a unit root?', Journal of Econometrics 54, 159178.

MacKinnon, J. (1994), 'Approximate asymptotic distribution functions for unitroot and cointegration tests', Journal of Business and Economic Statistics 12, 167-176.

MacKinnon, J. (1996), 'Numerical distribution functions for unit root and cointegration tests', Journal of Applied Econometrics 11, 601-618. 
MacKinnon, J. (2000), Computing numerical distribution functions in econometrics, in A. Pollard, D. Mewhort \& D. Weaver, eds, 'High Performance Computing Systems and Applications', Kluwer, Amsterdam, pp. 455-470.

Marsaglia, G. \& Tsang, W. W. (2000), 'The ziggurat method for generating random variables', Journal of Statistical Software 5(8), 1-7.

Osborn, D. R., Chui, A., Smith, J. P. \& Birchenhall, C. (1988), 'Seasonality and the order of integration for consumption', Oxford Bulletin of Economics and Statistics 50(4), 361-377.

Phillips, P. (1987), 'Time series regression with unit root', Econometrica 55(2), 277-301.

Phillips, P. \& Perron, P. (1988), ‘Testing for a unit root in time series regression', Biometrika 75, 335-46.

Saito, M. \& Matsumoto, M. (2008), SIMD-Oriented Fast Mersenne Twister: A 128-bit Pseudorandom Number Generator, in 'Monte Carlo and Quasi-Monte Carlo Methods', Springer-Verlag, Berlin. 
Table 1: Monte Carlo simulations for obtaining surface regressions and 95\% accuracy of some estimated quantiles.

\begin{tabular}{|c|c|c|c|c|c|c|}
\hline$s$ & $T$ & $p_{\max }$ & $N$ & $A_{0.10}$ & $A_{0.05}$ & $A_{0.01}$ \\
\hline 4 & $48,100,136,200,400$ & 8 & 50000 & 0.00053 & 0.00038 & 0.00017 \\
\hline 6 & $48,100,136,200,400$ & 12 & 50000 & 0.00053 & 0.00038 & 0.00017 \\
\hline 12 & $64,76,100,124,152,300,400,500$ & 12 & 10000 & 0,00118 & 0.00085 & 0.00039 \\
\hline 24 & $64,76,100,124,152,300,400,500$ & 12 & 10000 & 0,00118 & 0.00085 & 0.00039 \\
\hline \multicolumn{7}{|c|}{$s$ : periodicity } \\
\hline \multicolumn{7}{|c|}{$T$ : sample size } \\
\hline \multicolumn{7}{|c|}{$\begin{array}{l}p_{\max }: \text { maximum number of AR lags, so that for each } T \text {, models are simulated with } p=0,1 \ldots, p_{\max } \\
N \text { : number of replications }\end{array}$} \\
\hline
\end{tabular}




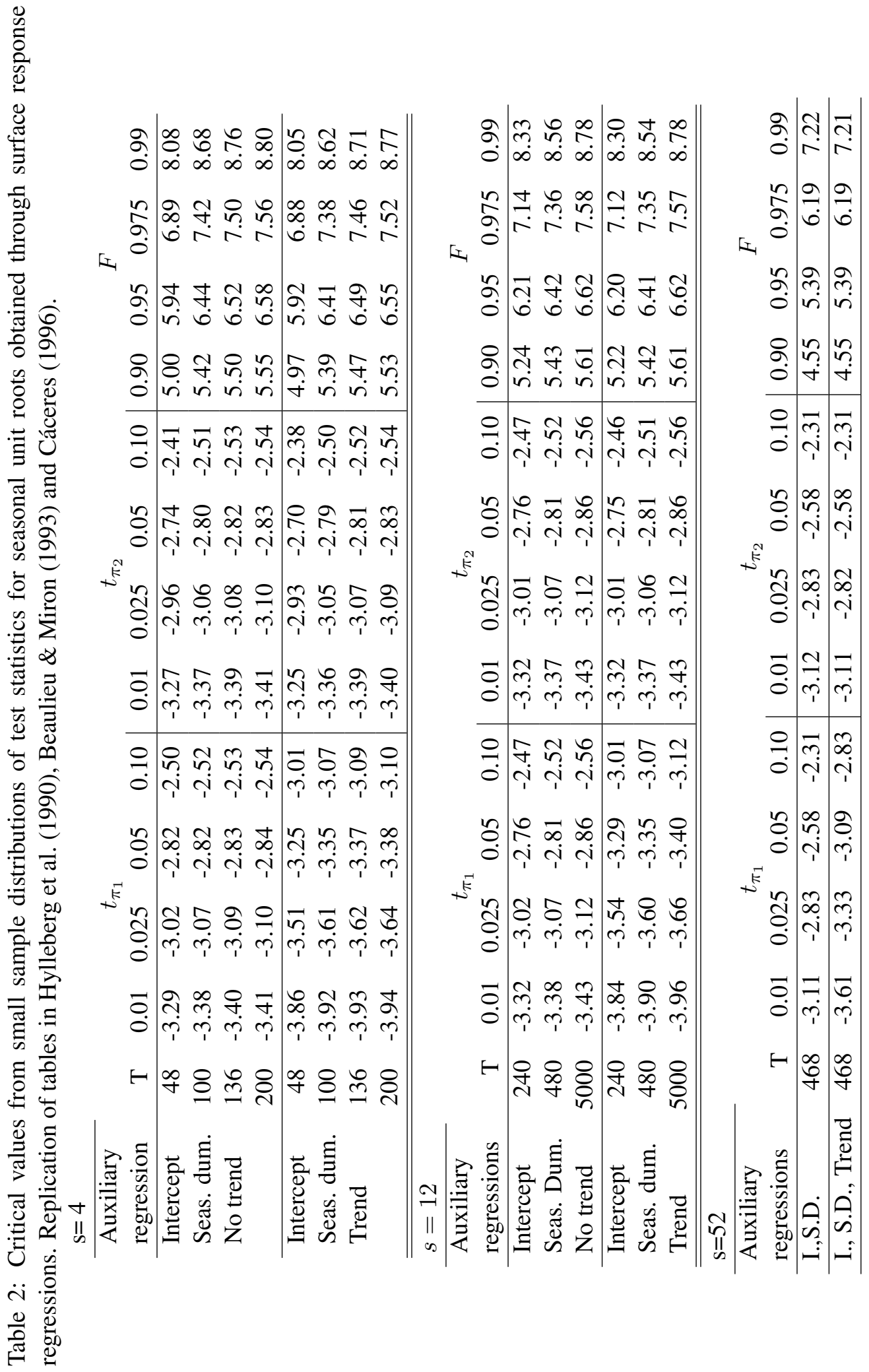


Table 3: Critical values from small sample distributions of test statistics for seasonal unit roots obtained through surface response regressions. Three types of daily observations.

\begin{tabular}{|c|c|c|c|c|c|c|c|c|c|}
\hline \multirow{2}{*}{$\begin{array}{l}\text { Auxiliary } \\
\text { regression }\end{array}$} & \multicolumn{5}{|c|}{$t_{\pi_{1}}$} & \multicolumn{4}{|c|}{$F$} \\
\hline & $\mathrm{T}$ & 0.01 & 0.025 & 0.05 & 0.10 & 0.90 & 0.95 & 0.975 & 0.99 \\
\hline Intercept & 48 & -3.26 & -2.97 & -2.76 & -2.44 & 4.86 & 5.81 & 6.76 & 7.97 \\
\hline Seas. dum. & 100 & -3.36 & -3.05 & -2.79 & -2.50 & 5.34 & 6.35 & 7.32 & 8.56 \\
\hline \multirow[t]{3}{*}{ No trend } & 136 & -3.38 & -3.07 & -2.81 & -2.52 & 5.43 & 6.45 & 7.42 & 8.66 \\
\hline & 200 & -3.40 & -3.09 & -2.83 & -2.53 & 5.50 & 6.52 & 7.49 & 8.73 \\
\hline & 400 & -3.42 & -3.11 & -2.84 & -2.55 & 5.57 & 6.59 & 7.56 & 8.78 \\
\hline Intercept & 48 & -3.82 & -3.47 & -3.21 & -2.95 & 4.83 & 5.78 & 6.74 & 7.93 \\
\hline Seas. dum. & 100 & -3.89 & -3.58 & -3.33 & -3.05 & 5.31 & 6.31 & 7.28 & 8.51 \\
\hline \multirow[t]{3}{*}{ Trend } & 136 & -3.91 & -3.60 & -3.35 & -3.07 & 5.40 & 6.41 & 7.38 & 8.62 \\
\hline & 200 & -3.92 & -3.62 & -3.37 & -3.09 & 5.48 & 6.50 & 7.46 & 8.70 \\
\hline & 400 & -3.94 & -3.64 & -3.39 & -3.11 & 5.56 & 6.58 & 7.54 & 8.76 \\
\hline
\end{tabular}

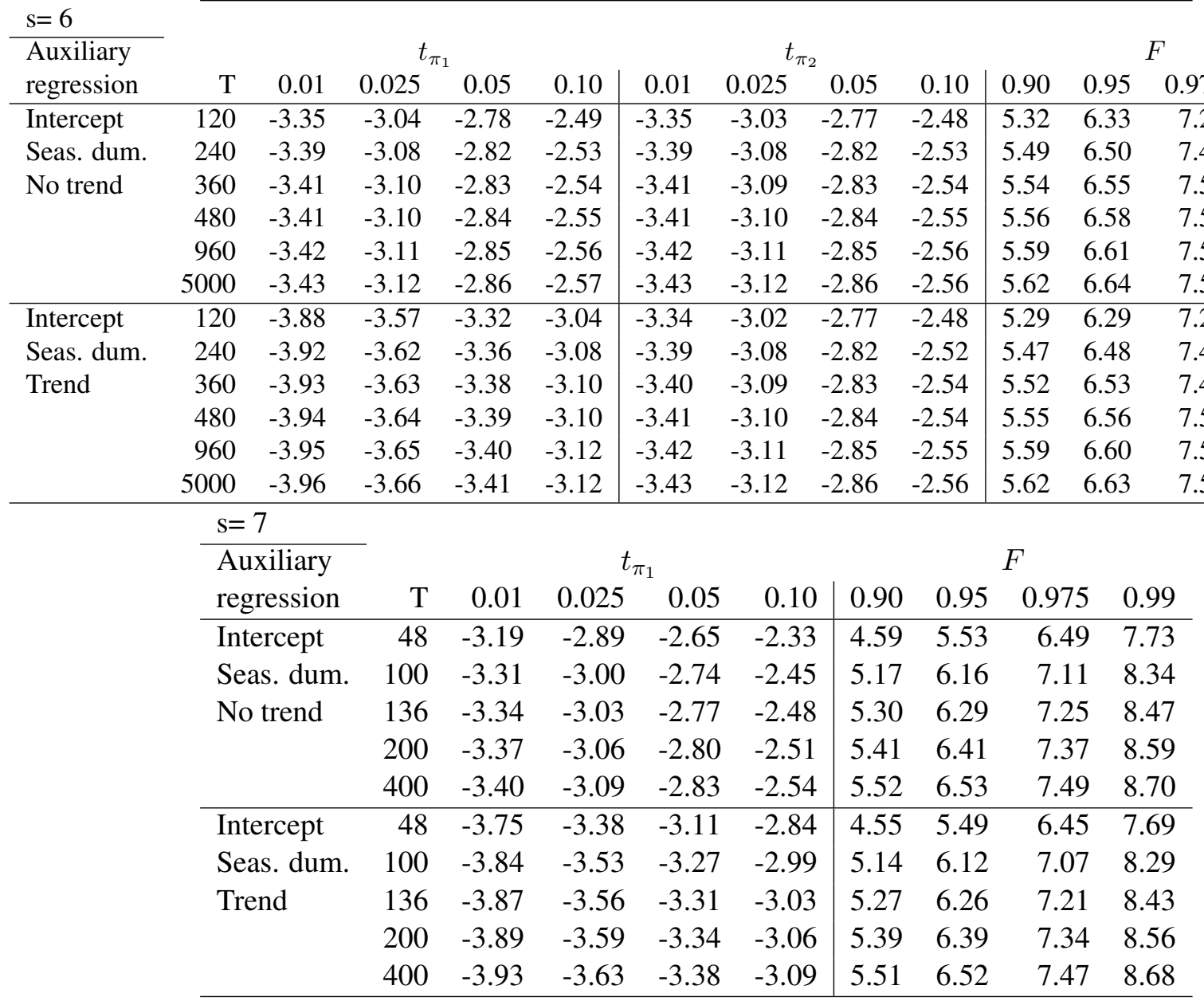




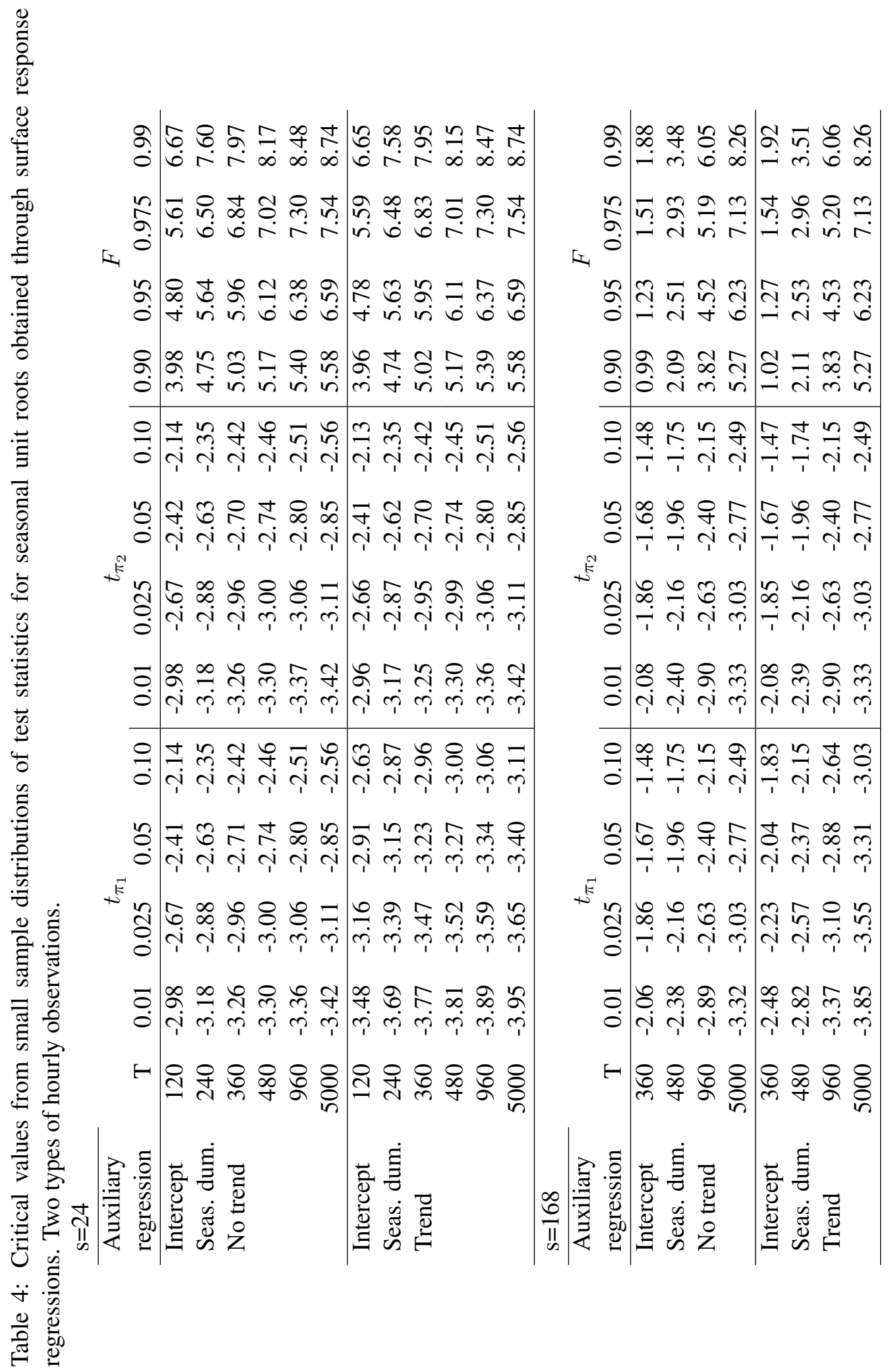




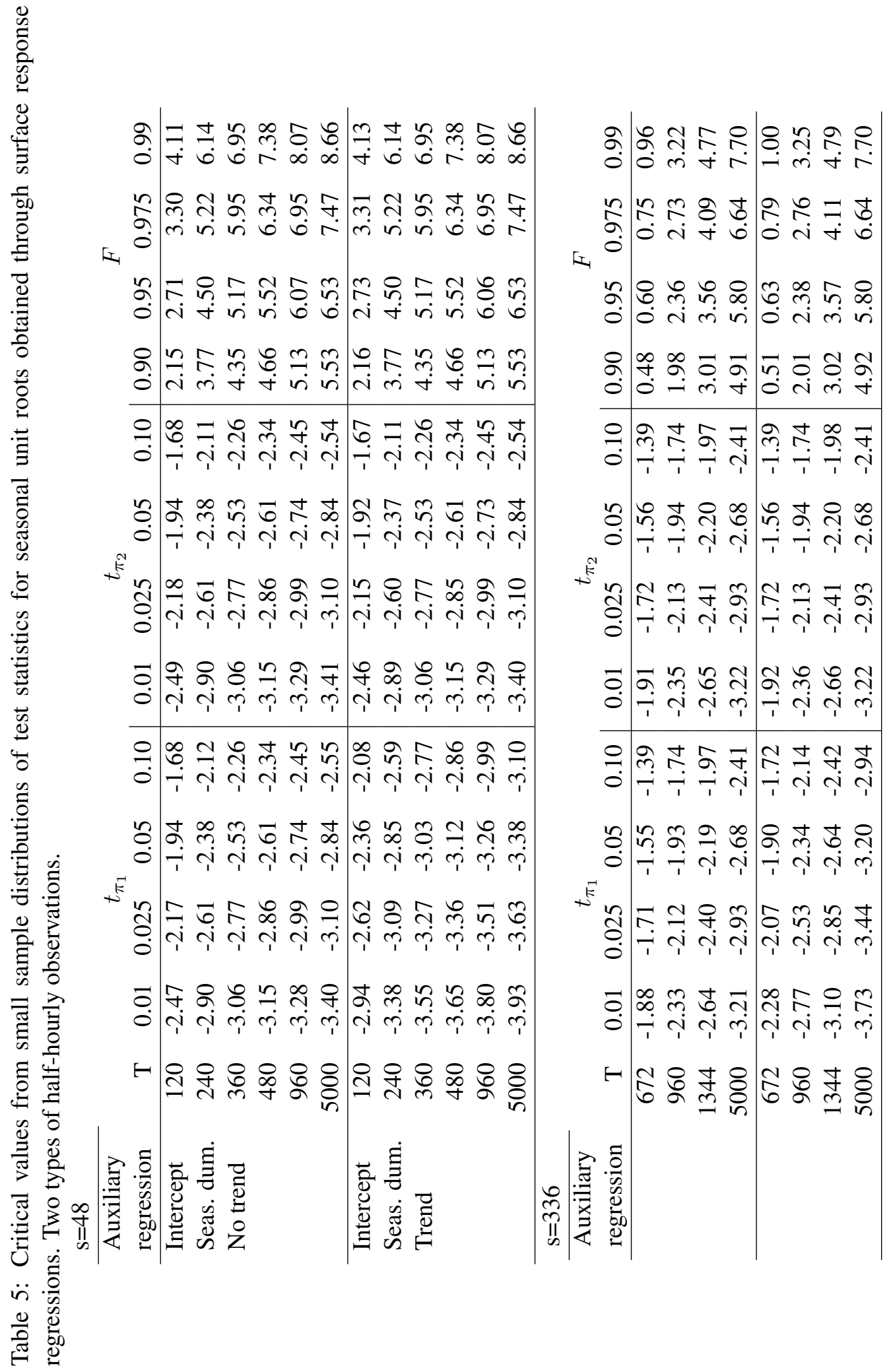

